\title{
Analytic dispersion relation of energetic particle driven geodesic acoustic modes and simulations with NEMORB
}

\author{
D. Zarzoso, A. Biancalani, A. Bottino, Ph. Lauber, E. Poli
}

Max-Planck Institut für Plasmaphysik, 85748 Garching, Germany

\author{
J.-B. Girardo, X. Garbet, R. J. Dumont \\ CEA, IRFM, F-13108 Saint-Paul-lez-Durance, France.
}

\begin{abstract}
Recent progress regarding the excitation of energetic-particle driven geodesic acoustic modes (EGAMs) in particle-in-cell simulations is presented in this paper. The exact dispersion relation with adiabatic electrons is derived and solved. The origin of the so-called EGAM is briefly analysed and we show that its nature changes, at least, with the safety factor. A simple expression for the GAM frequency modified in the presence of a small concentration of energetic particles is given in the fluid limit. We show that gyrokinetic simulations with NEMORB in the presence of adiabatic electrons are able to reproduce the analytic predictions. Also, different energy channels are analysed by means of dedicated energy diagnostics characterizing the wave-particle interaction. Finite Larmor radius and finite orbit width effects are studied regarding the excitation of geodesic acoustic modes, showing that these effects are likely to be negligible for sufficiently high concentration of energetic particles, but significant when approaching the threshold of excitation.
\end{abstract}




\section{Introduction}

The excitation of geodesic acoustic modes by energetic particles (EGAMs) has been predicted analytically [1, 2] and observed in both experiments [3, 4] and simulations [5]. There has been an increasing interest in these modes mainly due to the rather optimistic possibility of using them as an external knob to suppress turbulence [3]. First analysis of the interaction between energetic particles and turbulence via EGAMs has been conducted in electrostatic gyrokinetic simulations with adiabatic electrons [6]. In that work, an increase of turbulent transport was observed during the excitation of EGAMs. However, this apparently negative result was obtained together with the promising regulation of turbulence at the EGAM frequency. Both results could be explained by a strong interaction between EGAMs and ITG (Ion Temperature Gradient) modes via a parametric decay, following previous work done on standard GAMs [7]. Understanding the underlying mechanism is essential on the route towards the control of turbulence.

The EGAMs are axisymmetric modes and therefore driven mainly by the positiveness of the slope of the distribution function of ions at a certain position in velocity space, namely at $v_{\|}=q R \omega_{\mathrm{EGAM}}$ [5]. This is the reason why EGAMs must be described kinetically. The existence of these modes is based on the geodesic curvature of the magnetic field lines, as occurred with standard GAMs [8]. When coupling Vlasov and Poisson equations, one can derive a condition for the excitation of energetic-particle driven GAMs. This means that, strictly speaking, Ampère's law is not needed for the primary description of these modes. However, it is well-recognized that these modes are experimentally observed by means of diagnostics that detect the perturbations of the magnetic field, oscillating at the frequency $\omega_{E G A M}$. These perturbations are detected at the edge, whereas the mode is excited in the inner region. Therefore, it is obvious that a magnetic component of EGAMs must exist, with an eigenfunction extending from the position where the mode is excited to the position where the mode is detected.

Obtaining a complete kinetic and electromagnetic description of the excitation of EGAMs is the ultimate goal of including energetic particles in NEMORB[9, 10]. However, as an intermediate step, one needs to make sure not only that the code gives the correct excitation in some limits which can be verified, but also that we understand the physics of the excitation before going to more complex models. This is the motivation of the present work, which is divided into three parts. First, we derive the analytical dispersion relation in the presence of energetic particles, by assuming a linear electrostatic collisionless model with flat equilibrium profiles, treating the electrons as adiabatic, and neglecting finite Larmor radius (FLR) and finite orbit width (FOW) effects. A shifted Maxwellian distribution function in parallel velocity is adopted as distribution function for EP. The dispersion relation derived in this framework leads to the well-known frequency of GAMs in the limit of no energetic particles [11,5]. Second, we show results of numerical simulations of EGAMs performed with NEMORB, in the framework of an electrostatic linear collisionless model with electrons treated adiabatically, and verify that the code 
gives the expected results when going towards the analytic limit. Finally, analysis of the different energy channels characterizing the excitation of the mode is presented.

\section{Dispersion relation of EGAMs}

This section is a generalization of the analytic calculations performed in Ref. [5] to obtain the kinetic dispersion relation in the long wavelength limit, namely $k_{\perp} \rho_{i} \ll 1$, where $k_{\perp}$ is the perpendicular wave vector of the mode and $\rho_{i}$ is the ion Larmor radius. With respect to the calculations of Ref. [5], energetic particles are included and modelled by a double shifted Maxwellian

$$
F_{\text {eq,EP }}=\frac{1}{2} \frac{n_{\mathrm{EP}}}{\left(2 \pi T_{\mathrm{EP}} / m_{\mathrm{EP}}\right)^{3 / 2}} \mathrm{e}^{-\frac{\mu B}{T_{\mathrm{EP}}}}\left(\mathrm{e}^{-\frac{\left(v_{\|}-v_{0}\right)^{2}}{2 T_{\mathrm{EP}} / m_{\mathrm{EP}}}}+\mathrm{e}^{-\frac{\left(v_{\|}+v_{0}\right)^{2}}{2 T_{\mathrm{EP}} / m_{\mathrm{EP}}}}\right)
$$

whereas the thermal particles (also called bulk) are described by a centred Maxwellian

$$
F_{\text {eq, th }}=\frac{n_{\text {eq }}-n_{\mathrm{EP}}}{\left(2 \pi T_{\mathrm{th}} / m_{\mathrm{th}}\right)^{3 / 2}} \mathrm{e}^{-\frac{\mu B}{T_{\mathrm{th}}}} \mathrm{e}^{-\frac{v_{\|}^{2}}{2 T_{\mathrm{th}} / m_{\mathrm{th}}}}
$$

with $n_{\text {eq }}$ the total concentration and $n_{\mathrm{EP}}$ the concentration of energetic particles. The choice of a double shifted Maxwellian is made so that no parallel momentum is injected in the system. In addition, the parity of the distribution function in parallel velocity is essential to obtain the final expression of the dispersion relation.

In the following, the temperature $T_{\mathrm{EP}}$ describing the shifted Maxwellian for energetic particles is supposed to be the same as the temperature of the bulk $T_{\mathrm{th}}$, the mass of energetic particles $m_{\mathrm{EP}}$ is the same as the mass of thermal particles $m_{\mathrm{th}}$, the parallel

velocities $v_{\|}$and $v_{0}$ are normalized to the thermal velocity $v_{\mathrm{th}}=\sqrt{T_{\mathrm{th}} / m_{\mathrm{th}}}$ and rewritten as $\zeta$ and $\bar{\zeta}$, respectively. This calculation is supposed to shed some light on the way the energetic GAM is excited. By coupling the Vlasov equation and the quasi-neutrality equation and linearising around the equilibrium distribution function, one can obtain the following dispersion relation of GAMs in the presence of energetic particles (see Appendix A for the details of the derivation)

$$
\begin{aligned}
\mathcal{D}(\Omega)=\{1- & \left.\frac{1}{2}\left\langle\frac{\zeta^{4}+2 \zeta^{2}+2}{\Omega^{2}-\zeta^{2} / q^{2}}\right\rangle_{F_{\mathrm{eq}}}+\frac{1}{2}\left\langle\frac{\zeta^{4}+2 \zeta^{2}+2}{\Omega^{2}-\zeta^{2} / q^{2}}\right\rangle_{\mathrm{EP}}\right\} \\
& \times\left\{1+\tau-\langle 1\rangle_{\mathrm{EP}}-\left\langle\frac{\Omega}{\Omega-\zeta / q}\right\rangle_{F_{\mathrm{eq}}}+\left\langle\frac{\Omega}{\Omega-\zeta / q}\right\rangle_{\mathrm{EP}}\right\} \\
& -\frac{1}{2}\left\{\left\langle\frac{\zeta^{2}+1}{\Omega-\zeta / q}\right\rangle_{F_{\mathrm{eq}}}-\left\langle\frac{\zeta^{2}+1}{\Omega-\zeta / q}\right\rangle_{\mathrm{EP}}\right\}^{2}=0
\end{aligned}
$$

where $\Omega=\omega R / v_{\mathrm{th}}, R$ is the major radius of the tokamak, $\tau=T_{\mathrm{th}} / T_{e}$, with $T_{e}$ the 
electron temperature and the brackets represent the following averages in velocity space

$$
\begin{gathered}
\langle\cdots\rangle_{F_{\mathrm{eq}}}=\frac{1}{\sqrt{2 \pi}} \int d \zeta\{\cdots\} \sum_{\eta=0, \pm 1} N_{\eta} e^{\frac{(\zeta-\eta \bar{\zeta})^{2}}{2}} \\
\langle\cdots\rangle_{\mathrm{EP}}=\frac{1}{\sqrt{2 \pi}} \int d \zeta\{\cdots\} \frac{\bar{\zeta}}{\zeta} \sum_{\eta= \pm 1} \eta N_{\eta} e^{-\frac{(\zeta-\eta \bar{\zeta})^{2}}{2}}
\end{gathered}
$$

where $N_{0}=\frac{n_{\mathrm{eq}}-n_{\mathrm{EP}}}{n_{\mathrm{eq}}}, N_{ \pm 1}=\frac{n_{\mathrm{EP}}}{2 n_{\mathrm{eq}}}$ account for the concentrations of thermal and energetic particles, respectively. The average $\langle\cdots\rangle_{\mathrm{EP}}$ appears only if there are energetic particles in the system, which justifies the subscript $E P$, whereas the average $\langle\cdots\rangle_{F_{\mathrm{eq}}}$ is actually an average weighted by the equilibrium distribution function, containing both thermal and energetic particles and therefore does not vanish even in the absence of energetic particles. In particular, when $n_{\mathrm{EP}}=0$, the dispersion relation obtained in Ref. [5] is recovered

$$
\mathcal{D}(\chi)=\left\{\chi+q^{2} Z_{1}(\chi)\right\}\{1+\tau+\chi Z(\chi)\}-q^{2} \chi Z_{2}(\chi)^{2}=0
$$

where[12]

$$
\begin{aligned}
& Z_{1}(\chi)=\left(\chi^{4}+\chi^{2}+\frac{1}{2}\right) Z(\chi)+\chi\left(\chi^{2}+\frac{3}{2}\right) \\
& Z_{2}(\chi)=\chi+\left(\chi^{2}+\frac{1}{2}\right) Z(\chi)
\end{aligned}
$$

where the frequency $\Omega$ is renormalized as $\Omega \rightarrow \chi=q \Omega / \sqrt{2}$ and $Z(x)$ is the well-known plasma dispersion function. This dispersion relation corresponds to the one published in Ref. [13] in the limit of negligible diamagnetic effects (i.e. flat equilibrium profiles).

To solve the dispersion relation given by equation 3 , it is only necessary to compute the integrals

$$
\int d \zeta \frac{\zeta^{p}}{\Omega+\epsilon \zeta / q} e^{\frac{-(\zeta-\eta \bar{\zeta})^{2}}{2}}=2^{\frac{p}{2}} q \epsilon \sum_{k=0}^{p} \frac{p !}{k !(p-k) !}(\eta \bar{\chi})^{p-k}(-\epsilon)^{k+1} I_{k}(\chi+\epsilon \eta \bar{\chi})
$$

where $\epsilon= \pm 1, p=0,1,2,3,4, \eta=-1,0,1$ and the parallel velocity of energetic particles has been changed into $\bar{\chi}=\bar{\zeta} / \sqrt{2}$. Note that the functions $I_{k}$ are defined recursively as follows

$$
\begin{aligned}
& I_{k}(x)=x I_{k-1}(x)+\int u^{k-1} e^{-u^{2}} d u \\
& I_{0}(x)=\sqrt{\pi} Z(x)
\end{aligned}
$$

Therefore, the dispersion relation can be solved in a recursive way by computing numerically only the plasma dispersion function. This dispersion relation differs from the one published in Ref. [2] in the sense that we do not take into account the continuum damping due to equilibrium radial nonuniformities and we retain the Landau damping due to thermal species, which is essential for the existence of a threshold. Another difference is that we have decided to model energetic particles by a double shifted 
Maxwellian instead of using a slowing down distribution function. This is motivated by the fact that the former is analytically more tractable, yet retains the most essential physics element for the present study, i.e. the existence of a drive due to the wave-particle resonance. Whereas in our case this resonance occurs between the passing particles and the mode, in the case of a slowing-down distribution with a pitch-angle dependence the resonance occurs between the bounce motion of barely trapped particles and the mode. In the nonlinear phase these two distribution functions can exhibit different behaviours, as reported in Ref. [5], but nonlinear effects are not analysed in this paper, where only linear physics are retained, essential for the resonant drive. Our choice of double shifted Maxwellian is therefore motivated since it is analytically more tractable and allows us to perform analytic calculations in the fluid limit. In addition, it allows us to reveal and explain qualitatively some complex behaviour that might be observed in experiments in a simple way and detail how the mode is linearly excited.

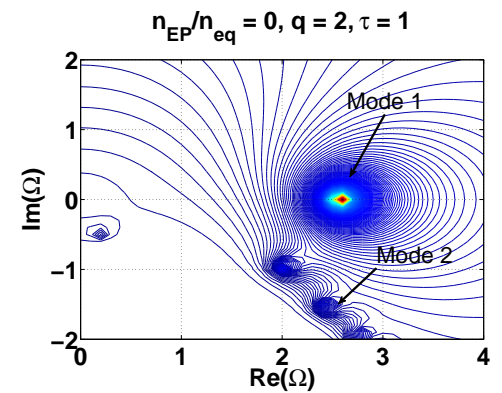

(a)

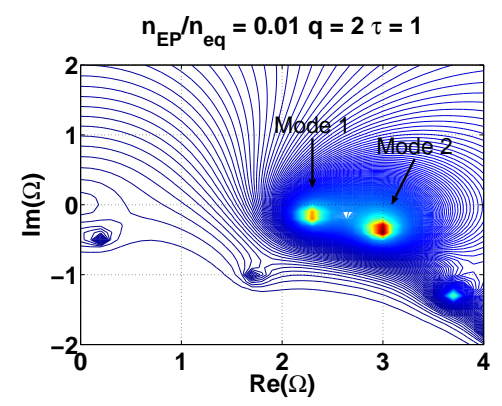

(d)

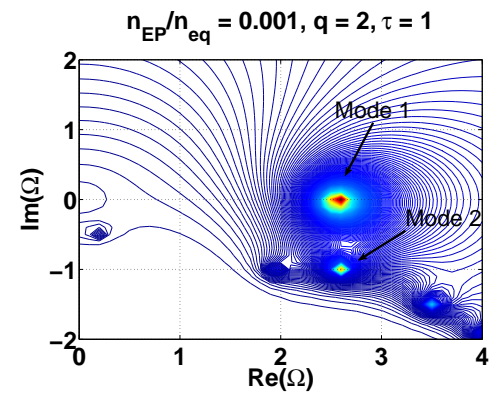

(b)

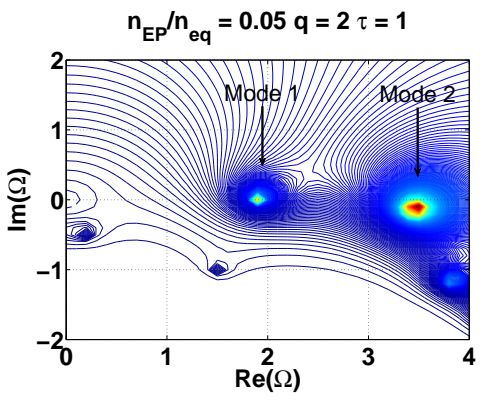

(e)

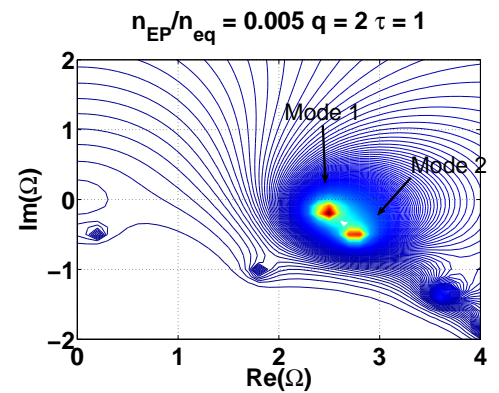

(c)

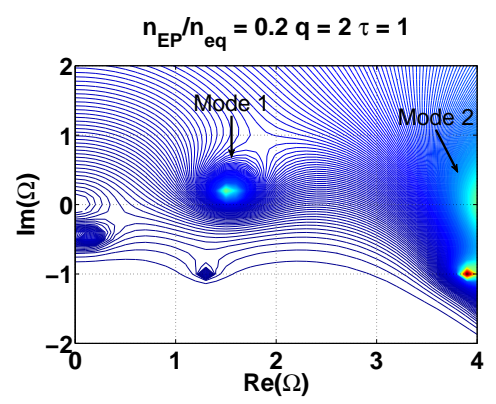

(f)

Figure 1: Evolution of the poles of $|\mathcal{D}(\Omega)|^{-1}$ with increasing energetic particle concentration with $q=2$.

The dispersion relation allows one to understand the evolution of the different zeros of $\mathcal{D}(\Omega)$ when increasing little by little the concentration of energetic particles, defined as the ratio of energetic particle density $n_{\mathrm{EP}}$ to total particle density $n_{\mathrm{eq}}$. This is especially useful to understand what the origin of the EGAM is. Is it really a new mode? Is it just a modification of the standard GAM? Figure 1 illustrates the evolution of the poles of $|\mathcal{D}(\Omega)|^{-1}$ for six different energetic particle concentrations and $q=2$. It is to be noted that this particular case of $q=2$ has been recently reported in ASDEX Upgrade 
[14]. It can be observed that the GAM, which is located initially at a frequency $\Omega=2.6$ and labeled as Mode 1 in figure 1a, is first damped when introducing a few energetic particles, as observed in figure 1c. At the same time, the mode labelled as Mode 2 in figure 1a exhibits an increase of frequency and an increase of growth rate (which remains always negative). The evolution of Mode 2 is accompanied by a decrease of the GAM frequency (Mode 1). This Mode 1 will eventually reach the real axis, as observed in figure 1e, and be excited with a further increase of energetic particle concentration as observed in figure $1 \mathrm{f}$. Therefore, the mode EGAM is born from the standard GAM whose frequency has been decreased with respect to the situation where only thermal particles exist. This picture changes when increasing the safety factor, for instance $q=3$. In that case we have observed that the Mode 1, which is initially the GAM, is not excited when introducing energetic particles and its frequency is increased. In that case, it is the Mode 2 which is excited, travelling on the complex plane towards decreasing frequencies, positive growth rates and therefore becoming the so-called EGAM. Note that this EGAM in the absence of energetic particles does exist, but is highly damped. It is contained in the branch of highly damped poles. This evolution is illustrated in figure 2. In conclusion, the mode called EGAM is not really a new mode, but an already existing mode excited in the presence of energetic particles. Depending on the values of $q$, it is born either from the initial GAM or from an initially highly damped mode. Further in-depth analysis about the dependence of the frequency and growth rate on the safety factor and other parameters like the energetic particle mass and energy is beyond the scope of this paper. The interested reader is encouraged to go through Ref. [15], where the theoretical dispersion relation is fully exploited in that direction, showing clearly the link between the energetic and the standard geodesic acoustic modes. Why the nature changes depending on the safety factor could be qualitatively explained by considering that the resonance occurs at the transit frequency, namely $\omega_{t}=v_{\|} / q R \approx v_{\text {res }} / q R$, where $v_{\text {res }}$ is the velocity of particles having a higher free energy from the point of view of the interaction with the mode, i.e. for particles exhibiting a maximum of $\partial_{E} F_{\text {eq. Starting }}$ from the situation without energetic particles, we have a GAM near the real axis and a branch of damped modes. If the parameter $v_{\text {res }} / q R$ is close to the GAM frequency, it is the standard GAM which will be excited. However, if that parameter is closer to the frequency of one of the initially damped modes, one of these modes will be excited, giving rise to a new branch. That is the reason why increasing the safety factor, and therefore decreasing $v_{\text {res }} / q R$ gives rise to the excitation of a mode coming from a branch different from the one of the standard GAM.

Finally, a simple expression for the GAM frequency modified in the presence of small concentration of energetic particles (GAM branch) can be obtained by taking the limit $q \Omega \gg 1$ (see Appendix B for details)

$$
\Omega_{\mathrm{GAM}, \mathrm{EP}} \approx \sqrt{\Omega_{\mathrm{GAM}}^{2}+\frac{3}{2} \frac{n_{\mathrm{EP}}}{n_{\mathrm{eq}}} \frac{v_{0}^{2}}{v_{\mathrm{th}}}}
$$

expressing the dependence of the GAM frequency on the energetic particle concentration 


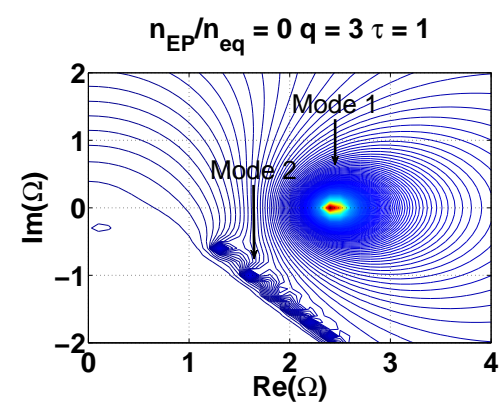

(a)

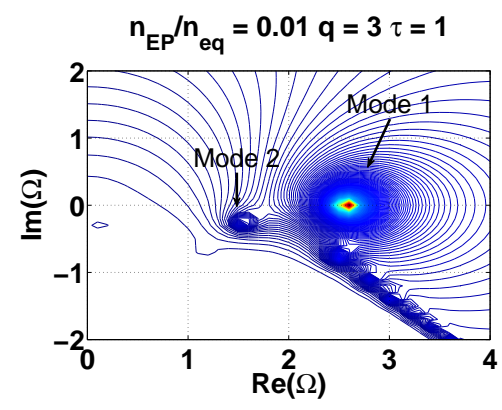

(d)

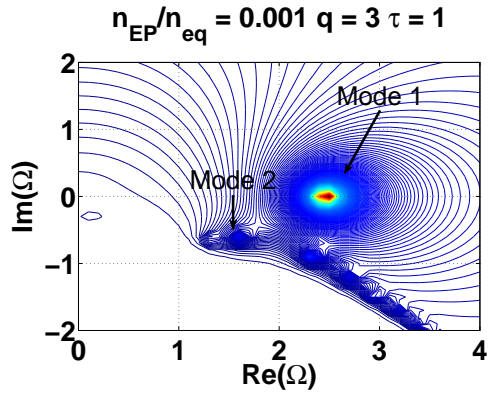

(b)

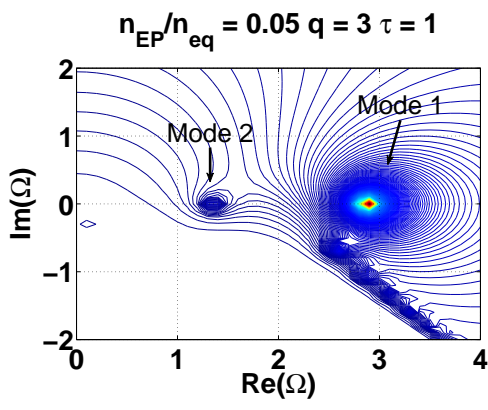

(e)

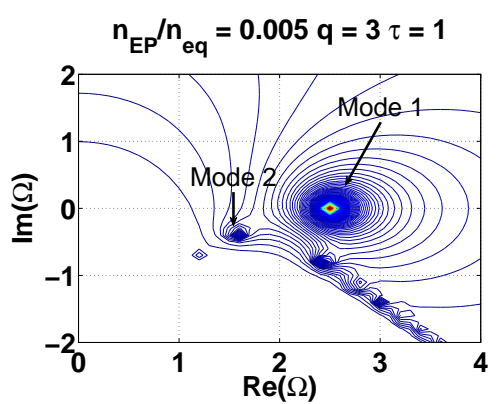

(c)

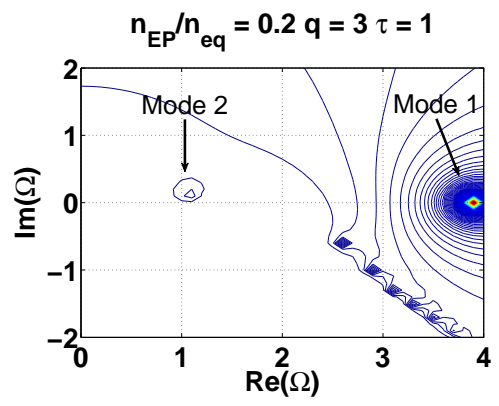

(f)

Figure 2: Evolution of the poles of $|\mathcal{D}(\Omega)|^{-1}$ with increasing energetic particle concentration with $q=3$.

and the mean velocity of the beam. Here $\Omega_{\text {GAM }}$ is the GAM frequency in the absence of energetic particles. Note that the limit $q \Omega \gg 1$ implies that either $q$, or $\Omega$ or both are high enough, which means that expression 10 can only describe the GAM branch for sufficiently high values of $q$. Note also that, although the previous expression introduces the dependence of the GAM frequency also on the mean velocity of the energetic particles, in this paper we keep this velocity as a fixed parameter and therefore we analyse only the impact of the energetic particle concentration.

\section{Gyrokinetic electrostatic simulations of EGAMs in Nemorb}

The dispersion relation that we gave in the previous section is compared with gyrokinetic simulations using the particle-in-cell (PIC) code NEMORB. In this section we first provide a brief description of the code. For further details, we refer the interested reader to already published material in Ref. [10], where the full model is described together with the discretization. After this brief introduction, we present the first benchmark of EGAM simulations when electrons exhibit an adiabatic response and show first evidence of the impact of FLR/FOW effects in the presence of energetic particles. 


\subsection{The gyrokinetic model and parameters of the simulations}

NEMORB solves the self-consistent and energy conserving nonlinear Vlasov-Maxwell system of equations in the modern gyrokinetic framework[16]. The gyrokinetic Vlasov equation for species $s$ in the absence of collisions and sources reads

$$
\frac{\mathrm{d} F_{s}}{\mathrm{~d} t}=\frac{\partial F_{s}}{\partial t}+\frac{\partial F_{s}}{\partial \mathbf{R}_{\mathbf{s}}} \dot{\mathbf{R}}_{s}+\frac{\partial F_{s}}{\partial p_{z, s}} \dot{p}_{z, s}=0
$$

where $\mathbf{R}_{s}$ is the gyrocentre position and $p_{z, s}$ is the canonical parallel momentum. The time derivatives of these two quantities are given by the equations of motion, which are actually the Euler-Lagrange equations, based on the Hamiltonian formulation, characterized by the function

$$
\mathcal{H}_{s}=\frac{p_{z, s}^{2}}{2 m_{s}}+\mu B+e_{s}\left(J_{0} \phi-\frac{p_{z, s}}{m_{s}} J_{0} A_{\|}\right)
$$

where $A_{\|}$is the parallel component of the perturbed magnetic potential, $m_{s}$ is the mass, $e_{s}$ the charge, $\mu$ the adiabatic invariant, $B$ the magnetic field, $J_{0}$ the gyroaverage operator and $\phi$ the electrostatic potential. To obtain the electrostatic potential, the Vlasov equation must be coupled to the quasineutrality condition, which in the long wave-length limit reads

$$
-\nabla_{\perp}\left(\sum_{s} \frac{m_{s} n_{0, s}}{e_{s} B^{2}}\right) \nabla_{\perp} \phi=\sum_{s} Z_{s} \delta n_{s}
$$

where $n_{0, s}$ is the equilibrium density of species $s, \delta n_{s}$ is the perturbed gyrocentre density and $Z_{s}$ is the charge of species $s$. In this paper we consider energetic particles in parallel velocity space, which means that the ion Larmor radius is not modified. Therefore, the long-wavelength limit for the polarization density term can be applied as in the case of thermal particles when one analyses the standard GAM. Nevertheless, in the polarization density, both a Padé approximation (as was done in GYSELA in Ref. [5]) and the long-wavelength approximation can be used in NEMORB and no significant difference has been observed.

In electrostatic simulations, i.e. in simulations where there are no magnetic perturbations $\left(A_{\|}=0\right)$, equations 11 and 13 represent a complete set of equations in order to obtain the solution. However, in the presence of magnetic perturbations, the Ampère's law must be retained and coupled to the Vlasov and quasineutrality equations. The linearized Ampère's law determines the evolution of the magnetic potential

$$
-\nabla_{\perp}^{2} A_{\|}=\mu_{0} \sum_{s} J_{\|, s}
$$

where $J_{\|, s}$ is the parallel current density linearized on the background distribution function $F_{\text {eq,s }}$. Because the present paper focuses on the electrostatic limit, Ampère's equation is not solved. In NEMORB, the electrons can be treated either as adiabatic, 
assuming $\delta n_{e} / n_{\mathrm{eq}} \propto e(\phi-\langle\phi\rangle) / T_{e}$, where $\langle\cdots\rangle$ is a flux-surface average, or kinetic, following a drift-kinetic equation, i.e. a gyrokinetic Vlasov equation with $J_{0}=1$. In addition, when electrons are treated kinetically, this can be done by considering either only trapped kinetic electrons and adiabatic passing electrons, or by considering that both trapped and passing electron populations exhibit a kinetic response.

For the simulations carried out in this paper, we use flat equilibrium profiles, and perform collisionless, electrostatic, linear simulations with adiabatic electrons. Therefore, there is no magnetic shear $(s=0)$ and no gradient driven instabilities like ion-temperature-gradient (ITG) turbulence. The ion Larmor radius normalized to the minor radius is $\rho_{*}=1 / 64$, unless another value is explicitly given. Ion and electron temperatures are the same, i.e. $\tau=1$. The parameters for the energetic particle distribution function are $\bar{\zeta}=4, T_{\mathrm{EP}}=T_{\mathrm{th}}$ and $m_{\mathrm{EP}}=m_{\mathrm{D}}$, where $m_{\mathrm{D}}$ is the Deuterium mass, used here as the thermal species. The number of markers used for each species is $10^{7}$ and we consider one single toroidal mode $n=0$, i.e. only axisymmetric modes. The number of radial and toroidal points is $N_{r}=64$ and $N_{\varphi}=4$, respectively. We use Neumann boundary conditions for the electrostatic potential $\partial_{r} \phi=0$ at $r=r_{\min }$, whereas zero Dirichlet boundary conditions are imposed at $r=r_{\max }$ for every time step. We use an initial perturbation for the electrostatic potential

$\phi(r, t=0) \simeq \delta \phi(0) \cos \left(\frac{\pi r}{2 r_{\max }}\right)$, where $\delta \phi(0)$ is the initial amplitude perturbation. The radial simulation domain is $r \in\left[r_{\min }, r_{\max }\right]$, where $r_{\min }=0$ and $r_{\max }=a$. The time step is set to $\Delta t=10$, in thermal ion cyclotron units. This is enough when the parallel electron dynamics does not need to be resolved, which is actually the case for adiabatic electrons. Convergence tests have been performed on the number of markers, time step and radial resolution. The results of these convergence tests are not shown here for the sake of brevity and we have decided to use the numerical parameters in order to optimize the CPU time and get quantitative meaningful physical results.

\subsection{Comparison of theoretical dispersion relation with gyrokinetic simulations and impact of FLR/FOW effects}

In this subsection, we compare our analytic theory with simulations using NEMORB. Regarding the Vlasov equation, we decompose the distribution function into an analytically known background $F_{\text {eq }}$ and a perturbed $\delta F$ distribution functions and solve an equation only on $\delta F$

$$
\frac{\mathrm{d} \delta F}{\mathrm{~d} t}=-\frac{\mathrm{d} F_{\mathrm{eq}}}{\mathrm{d} t}
$$

where $F_{\text {eq }}$ is written as a function of the energy $\mathcal{H}$, the adiabatic invariant $\mu$ and the position $\mathbf{R}$ and $\mathrm{d} / \mathrm{d} t$ is the convective derivative. Note that so far we have not made any assumption on the ordering of the perturbed distribution function. This means that it does not necessarily represents a small ordering with respect to the background distribution function, i.e. $\delta F$ can contain a contribution of the same order as $F_{\text {eq }}$ and other contributions of smaller order, which are typically referred to in the literature as 
perturbations. However, since our theory is linear, we need to perform linear simulations, which means that we need to assume that $\delta F$ is of first order in perturbations and does not contain any zero-th order contribution. The time derivative of the background reads

$$
\frac{\mathrm{d} F_{\text {eq }}}{\mathrm{d} t}=\nabla F_{\text {eq }} \cdot \dot{\mathbf{R}}
$$

where we have used the fact that $\mathcal{H}$ is a constant of motion on the unperturbed trajectories and $\mu$ is an adiabatic invariant. If we assume that the only dependence on $\mathbf{R}$ comes from the electrostatic potential, i.e, we assume flat profiles and consider no radial dependence of $B$, the background distribution function can be explicitly written as $F_{\text {eq }}(\mathcal{H}-e \phi, \mu)$, where we have already included the spatial dependence in the electrostatic potential. Therefore, the time derivative reads

$$
\frac{\mathrm{d} F_{\mathrm{eq}}}{\mathrm{d} t}=\partial_{E} F_{\mathrm{eq}}(-e \nabla \phi \cdot \dot{\mathbf{R}})
$$

where $E=\mathcal{H}-e \phi$. As far as energetic particles are concerned, the background distribution function can be written, assuming that $T_{\mathrm{EP}}=T_{\mathrm{th}}$, as $F_{\mathrm{eq}, \mathrm{EP}}=$ $F_{\text {eq, th }}(E) f(E, \mu)$ (see equations 1 and 2), where the factor $f$ represents the modification of a local Maxwellian $F_{\text {eq,th }}$ due to the presence of energetic particles. Therefore, the time derivative of the background for the energetic particles reads

$$
\frac{\mathrm{d} F_{\text {eq,EP }}}{\mathrm{d} t}=\frac{1}{T_{\mathrm{th}}} F_{\text {eq,EP }}\left(1-T_{\mathrm{th}} \partial_{E} \log f\right) e \nabla \phi \cdot \dot{\mathbf{R}}
$$

and therefore, the Vlasov equation for energetic particles reads

$$
\frac{\mathrm{d} \delta F_{\mathrm{EP}}}{\mathrm{d} t}=-\frac{1}{T_{\mathrm{th}}} F_{\text {eq,EP }}\left(1-T_{\text {th }} \partial_{E} \log f\right) e \nabla \phi \cdot \dot{\mathbf{R}}
$$

We can decompose $\phi$ and $\dot{\mathbf{R}}$ into zero-th order and first order terms: $\phi=\phi^{(1)}$ and $\dot{\mathbf{R}}=\dot{\mathbf{R}}^{(0)}+\dot{\mathbf{R}}^{(1)}$. We have considered that the amplitude of the electrostatic potential $\delta \phi$ gives the order of the perturbed quantities, i.e. $\left\|\phi^{(1)}\right\|=\mathcal{O}(|\delta \phi|)$. In this way, the linearization of this equation is straightforward

$$
\left.\frac{\mathrm{d} \delta F_{\mathrm{EP}}}{\mathrm{d} t}\right|_{\text {lin }}=-\frac{1}{T_{\mathrm{th}}} F_{\text {eq,EP }}\left(1-T_{\mathrm{th}} \partial_{E} \log f\right) e \nabla \phi^{(1)} \cdot \dot{\mathbf{R}}^{(0)}
$$

In this equation we have not considered the spatial dependence of the magnetic field. This will add an extra term in the presence of energetic particles due to the gradient of $B$. The Vlasov equation is in this case written as follows

$$
\frac{\mathrm{d} \delta F_{\mathrm{EP}}}{\mathrm{d} t}=-\frac{1}{T_{\mathrm{th}}} F_{\mathrm{eq}, \mathrm{EP}} e \nabla \phi \cdot \dot{\mathbf{R}}+F_{\mathrm{eq}, \mathrm{EP}} \partial_{E} \log f(e \nabla \phi+\mu \nabla B) \cdot \dot{\mathbf{R}}
$$

Linearizing equation 21 leads to the following expression

$$
\left.\frac{\mathrm{d} \delta F_{\mathrm{EP}}}{\mathrm{d} t}\right|_{\mathrm{lin}}=-\frac{1}{T_{\mathrm{th}}} F_{\mathrm{eq}, \mathrm{EP}} e \nabla \phi^{(1)} \cdot \dot{\mathbf{R}}^{(0)}+F_{\mathrm{eq}, \mathrm{EP}} \partial_{E} \log f\left(e \nabla \phi^{(1)} \cdot \dot{\mathbf{R}}^{(0)}+\mu \nabla B \cdot \dot{\mathbf{R}}^{(1)}\right)
$$


where the zero-th order particle velocity is the parallel velocity and curvature and $\nabla B$ drift velocities $\dot{\mathbf{R}}^{(0)}=v_{\|} \mathbf{b}+\mathbf{v}_{D}$ and the first order velocity is the $E \times B$ drift $\dot{\mathbf{R}}^{(1)}=-B^{-1} \nabla \phi^{(1)} \times \mathbf{b}$. In this linearized equation we have not included the term proportional to $\mu \nabla B \cdot \dot{\mathbf{R}}^{(0)}$ since it is a zero-th order term. Note that, by doing this, we are implicitly considering only the part of the perturbed distribution function which satisfies the ordering $\left\|\delta F_{\mathrm{EP}}\right\|=\mathcal{O}(|\delta \phi|)$ and therefore assuming that $\delta F_{\mathrm{EP}}$ does not contribute to the evolution of the equilibrium. In order words, this term expresses the fact that the total distribution function $F_{\mathrm{eq}, \mathrm{EP}}+\delta F_{\mathrm{EP}}$ does not depend on the motion invariants and might therefore evolve even in the absence of an excited mode due to a zero-th order term in $\delta F_{\mathrm{EP}}$. Leaving out this term is legitimate in a linearized system where a distinction between equilibrium and perturbations is needed.

The $\mu \nabla B \cdot \dot{\mathbf{R}}^{(1)}$ term is expected to play little role regarding the excitation of a mode scaling as $\phi^{(1)} \sim \delta \phi \sin \theta$. The reason is the following. For a $\sin \theta$-like mode, the gradient scales as $\nabla \phi^{(1)} \sim \delta \phi \cos \theta \mathbf{e}_{\theta}$, with $\mathbf{e}_{\theta}$ the unit vector in the poloidal direction. Therefore $\dot{\mathbf{R}}^{(1)-1} \delta \phi \cos \theta \mathbf{e}_{r}$, with $\mathbf{e}_{r}$ the unit vector in the radial direction. In the limit of large aspect ratio, $\nabla B \sim \varepsilon B\left(\cos \theta \mathbf{e}_{r}+\sin \theta \mathbf{e}_{\theta}\right)$, with $\varepsilon$ the inverse of the aspect ratio. Therefore, the first order particle velocity contributes as $\nabla B \cdot \dot{\mathbf{R}}^{(1)} \sim \varepsilon \delta \phi(1+\cos 2 \theta) / 2$. The effect of the $\mu \nabla B$ term is thus of the order $\sim \varepsilon \delta \phi / 2$ and therefore small compared to the perturbations in the large aspect ratio limit. In addition, it does not contain any $\cos \theta$ mode. In conclusion, the $\mu \nabla B$ term is expected to play little role in the energy transfer between particles and a $\sin \theta$-like mode. In the framework of numerical verification, and only in this section, this term is not taken into account for the evolution of the background distribution function since it was neglected in the derivation of the analytic dispersion relation. Note however that this term is kept for the calculation of the trajectories, which is essential for the energy diagnostic analysis that is presented in section 4. We will see later that this term is found to contribute little to the linear growth rate.

The Vlasov equation is coupled to the quasi-neutrality equation, under the assumption of adiabatic electrons. In this case, the equations of motion will be solved only for the ions and the quasi-neutrality equation reads

$$
\frac{e n_{0}}{T_{e}}(\phi-\langle\phi\rangle)-\nabla_{\perp} \cdot\left(\sum_{s \neq e} \frac{m_{s} n_{0, s}}{e_{s} B^{2}}\right) \nabla_{\perp} \phi=\sum_{s \neq e} Z_{s} \delta n_{s}
$$

where the first term in the left-hand side represents the adiabatic response of electrons.

Let us recall that NemorB has already proved its capability to simulate EGAMs with adiabatic electrons. First benchmarks against the electrostatic simulations performed with the gyrokinetic code GYSELA [5] have been successfully made and published very recently [17]. The aim of this section is twofold. First, to provide quantitative verification of the multi-species version of the code when introducing a non Maxwellian distribution function. Second, prove that the FLR/FOW effects depend on the concentration of energetic particles. 
We perform a scan in energetic particle concentration for two values of $q$, namely $q=2$ and $q=3$. The results are shown in figures 3 and 4 . The values of the frequency are obtained by performing a Fourier transform in time of the $m=1$ component of the electrostatic potential at the mid radial position. The growth rates are measured by a linear regression of the mode $m=1$ at the mid radial position in logarithmic scale.

For both cases $q=2$ and $q=3$, the solid lines correspond to the frequency obtained with Nemorb, whereas the dashed lines represent the frequency predicted with the dispersion relation. We observe a very good agreement between the predicted values and the numerical observations for both quantities. As predicted from the theory, in the case $q=2$ the excited mode is born from the original GAM. This can be clearly observed from the continuity of the frequency and growth rate with respect to the energy particle concentration. The frequency of the excited mode is slightly decreased when increasing the safety factor from $q=2$ to $q=3$. This decrease in the frequency can also be obtained from the theoretical approach detailed in the previous section.

In the case $q=3$, as predicted in the previous section, the excited mode comes from an initially highly damped mode in the absence of energetic particles. Therefore, the EGAM belongs to a different branch from the GAM. This has been observed numerically as depicted in figure 4. In blue we represent the GAM branch, which is the only observable mode in the absence of energetic particles due to the smaller damping rate with respect to the other modes. When we increase the concentration of energetic particles, the GAM frequency is increased, in qualitative agreement with the fluid prediction given by equation 10, and the EGAM branch, represented by the red line, starts being observed, though damped. Increasing the energetic particle concentration leads to the existence of the EGAM branch only, while the GAM is completely damped.

We observe a good quantitative agreement, except for three values of the energetic particle concentration, namely $n_{\mathrm{EP}} / n_{\mathrm{th}}=[0,0.05,0.1]$. The case $n_{\mathrm{EP}} / n_{\mathrm{th}}=0$ corresponds to the standard GAM. It is well-known that for the standard GAM the ion Landau damping rate strongly depends on FLR/FOW effects via $k_{\perp} \rho_{i}$ and the safety factor $q[18]$ as was recently observed in NEMORB simulations[17]. Therefore, one might expect that the disagreement observed for $n_{\mathrm{EP}} / n_{\mathrm{th}}=[0.05,0.1]$ is also due to FLR/FOW effects. Regarding the FOW effects, this seems in agreement with the fact that for $q=2$, the numerical results match the theoretical predictions. As far as the FLR effects are concerned, this can be analysed by decreasing $\rho_{*}=\rho_{i} / a$. We have therefore run simulations for those energetic particle concentrations at $\rho_{*}=1 / 128$ and measured the growth rate. These new simulations are represented in figure $4 \mathrm{~b}$ by red asterisks, showing that the simulations converge towards the analytic theory in the limit $k_{\perp} \rho_{i} \rightarrow 0$. Further simulations at $\rho_{*}=1 / 256$ have also been performed and the growth rate does not change significantly with respect to the case $\rho_{*}=1 / 128$. We can therefore conclude that NEMORB is able to reproduce quantitatively theoretical predictions in the limit of adiabatic electrons and without FLR/FOW effects. In addition, a simulation using $n_{\mathrm{EP}} / n_{\mathrm{th}}=0.3$ at $q=3$ and $\rho_{*}=1 / 128$ has been performed and there is no significant difference with respect to the case $\rho_{*}=1 / 64$, meaning that FLR/FOW effects are less 


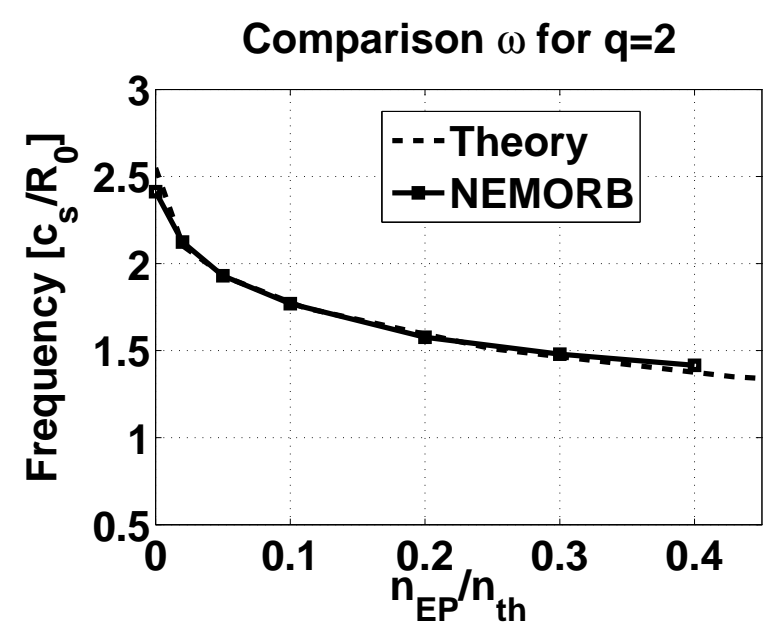

(a)

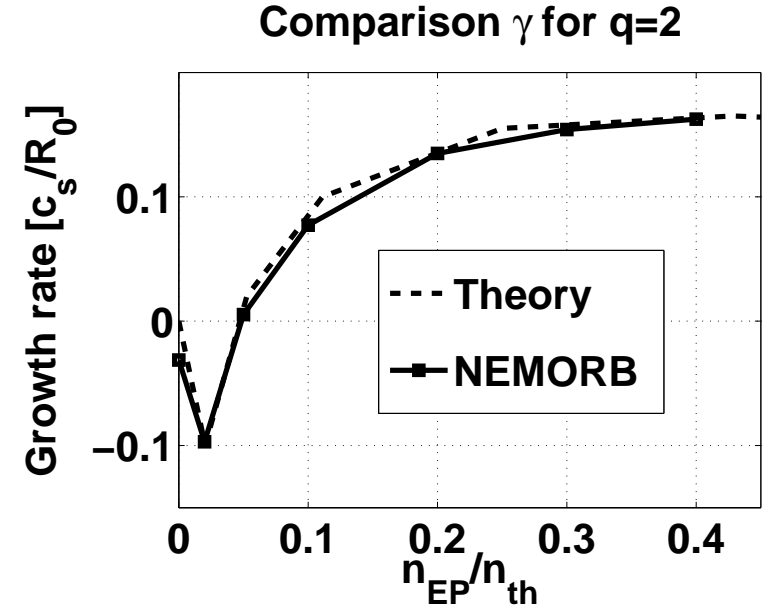

(b)

Figure 3: Comparison between the frequency and the growth/damping rates between NEMORB simulations with adiabatic electrons and predicted values from the dispersion relation for $q=2$.

pronounced when adding more energetic particles in the system.

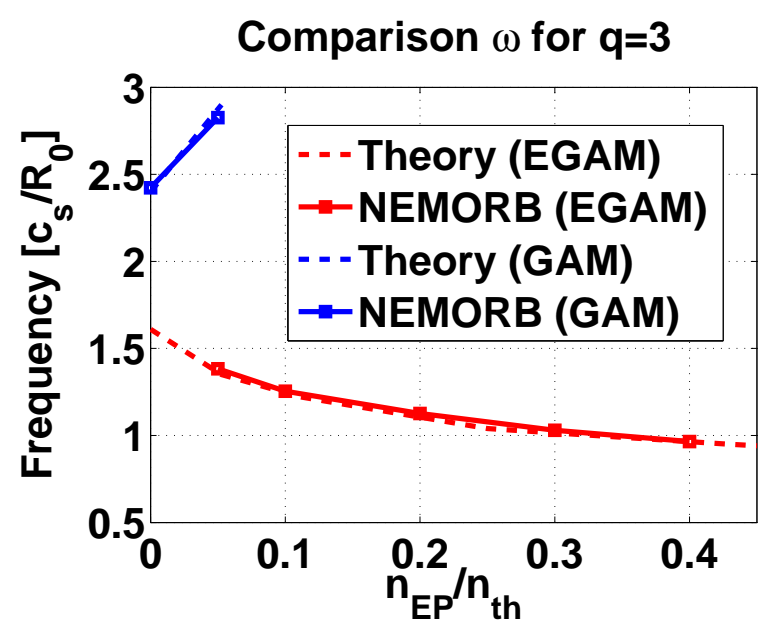

(a)

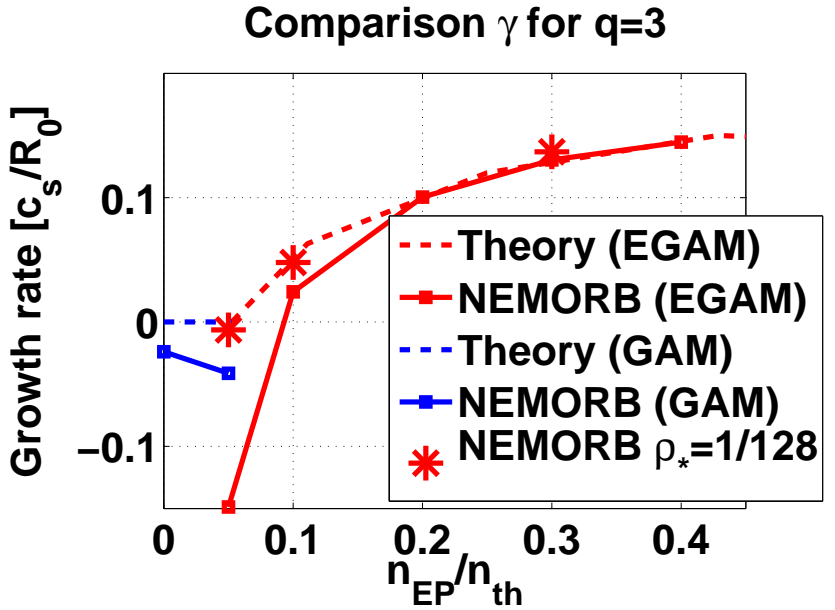

(b)

Figure 4: Comparison between the frequency and the growth/damping rates between NEMORB simulations with adiabatic electrons and predicted values from the dispersion relation for $q=3$.

In order to know to what extent the $\mu \nabla B$ term that was neglected in the linear simulations plays a role for the excitation of the mode, nonlinear simulations with the $\mu \nabla B$ term (or mirror term) have been performed and compared to the linear simulations in the absence of this mirror term. Figure 5 illustrates, in logarithmic scale, the time evolution of the $\sin \theta$ component of the electrostatic potential at the mid radial position for a concentration of energetic particles $n_{\mathrm{EP}} / n_{\mathrm{th}}=0.3$ for $q=2$ with (solid red line) and without (dashed blue line) mirror effects. It can be observed that the growth rate 
during the linear phase does not change significantly. The main difference between these two simulations is the initial state of the system, due to the fact that the presence of $\mu \nabla B$ represents a modification of the distribution function and therefore a different initial condition for the excited mode.

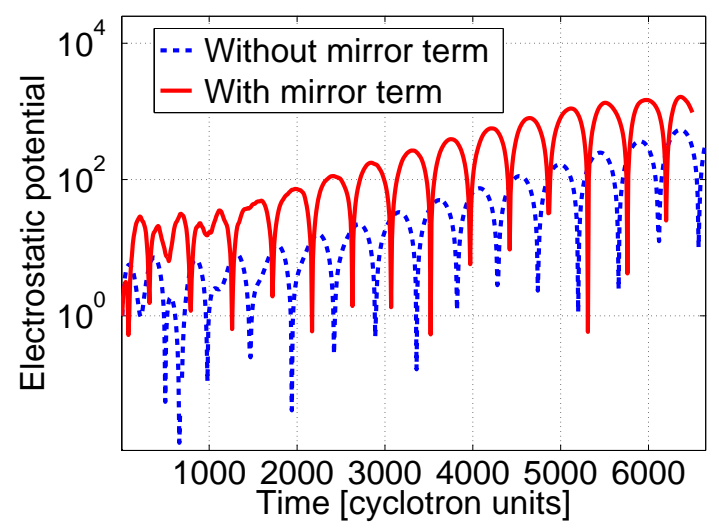

Figure 5: Comparison of linear growth phase between two simulations at $q=2$ and $n_{\mathrm{EP}} / n_{\mathrm{th}}=0.3$ with (solid red line) and without (dashed blue line) $\mu \nabla B$ term.

\section{Energy diagnostics}

More in-depth analysis can be done by examining the energy which is directly transferred from particles to the mode. In that respect, a multi-species code like NEMORB represents an optimal tool to determine the energy which is transferred from each species to the mode. The energy transfer is quantified by means of the expression

$$
\frac{\mathrm{d} E_{\text {kin }, s}}{\mathrm{~d} t}=\int \mathrm{d}^{3} \mathbf{x} \mathbf{J}_{s} \cdot \mathbf{E}
$$

which represents the increase or decrease of kinetic energy of species $s$ interacting with the electric field $\mathbf{E}$. This quantity is obtained in NEMORB as follows

$$
\left.\frac{\mathrm{d} E_{\text {kin }, s}}{\mathrm{~d} t}\right|_{\text {Nemorb }}=-q_{s} \int \frac{\mathrm{d} \mathbf{R}}{\mathrm{d} t} \cdot \nabla\langle\phi\rangle \delta F_{s} B_{\|}^{*} \mathrm{~d}^{3} \mathbf{R} \mathrm{d} v_{\|} \mathrm{d} \mu \mathrm{d} \alpha
$$

where $\langle\phi\rangle$ is the gyro-averaged electrostatic potential, $\alpha$ is the gyro-angle and $B_{\|}^{*} \mathrm{~d}^{3} \mathbf{R} \mathrm{d} v_{\|} \mathrm{d} \mu \mathrm{d} \alpha$ is the phase-space volume element. The expression 25 , in the absence of collisions between species, must equal the decrease or decrease of energy of the electric field due to each species $E_{f, s}$, i.e.[19]

$$
\left.\frac{\mathrm{d} E_{\text {kin }, s}}{\mathrm{~d} t}\right|_{\text {Nemorb }}=-\left.\frac{\mathrm{d} E_{\mathrm{f}, s}}{\mathrm{~d} t}\right|_{\text {Nemorb }}
$$

where the field energy is defined to the lowest order in perturbations as [20,21]

$$
E_{\mathrm{f}, s}(t)=\frac{q_{s}}{2} \int\left[\left\langle n_{s}(\mathbf{R}, t)\right\rangle-\left\langle n_{0, s}(\mathbf{R})\right\rangle\right] \phi(\mathbf{R}, t) \mathrm{d}^{3} \mathbf{R}
$$


and the brackets $\langle\cdots\rangle$ represent in this case a gyro-average. The total energy of the field can then be obtained simply by summation over all the species, i.e.

$$
E_{\mathrm{f}}=\sum_{s} E_{\mathrm{f}, s}
$$

The time derivative of the kinetic energy, i.e. expression 25, can be decomposed into different contributions of the guiding centre velocity, namely the parallel velocity, the curvature drift and the $\mu \nabla B$ drift (we neglect the contribution of the $E \times B$ drift since it represents a second order term)

$$
\left.\frac{\mathrm{d} E_{\text {kin }, s}}{\mathrm{~d} t}\right|_{\text {NemorB }}=q_{s} \int\left(v_{\|} \mathbf{b}+\frac{\mathbf{B}}{q_{s} B^{2}} \times \mu_{s} \nabla B+m_{s} v_{\|}^{2} \frac{\mathbf{B}}{q_{s} B^{2}} \times \mathbf{b} \cdot \nabla \mathbf{b}\right) \cdot\langle\mathbf{E}\rangle \delta F_{s} B_{\|}^{*} \mathrm{~d}^{3} \mathbf{R} \mathrm{d} v_{\|} \mathrm{d} \mu \mathrm{d} \alpha
$$

Energy diagnostics are essential to verify that the energy conservation given by equation 26 is satisfied in our simulations when introducing energetic particles as a second species. As an example, we quantify the energy conservation for an energetic particle concentration $n_{\mathrm{EP}} / n_{\mathrm{eq}}=0.2$ and $q=2$. This is shown in figure 6 . On the one hand, figure 6 a represents the time evolution of the sum over all species of the time derivative of the kinetic energy (thin solid line) and the field energy (thick dashed line). On the other hand, figure $6 \mathrm{~b}$ shows the relative error, which is found to be less than $5 \%$.

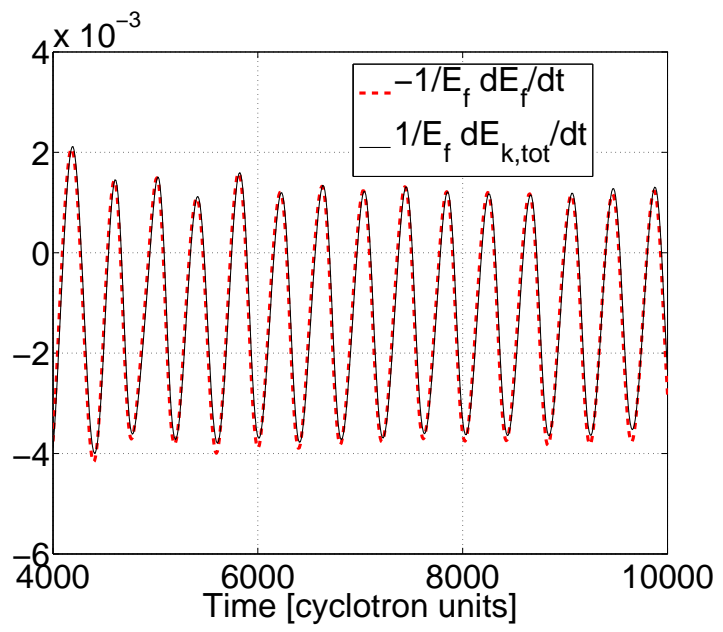

(a)

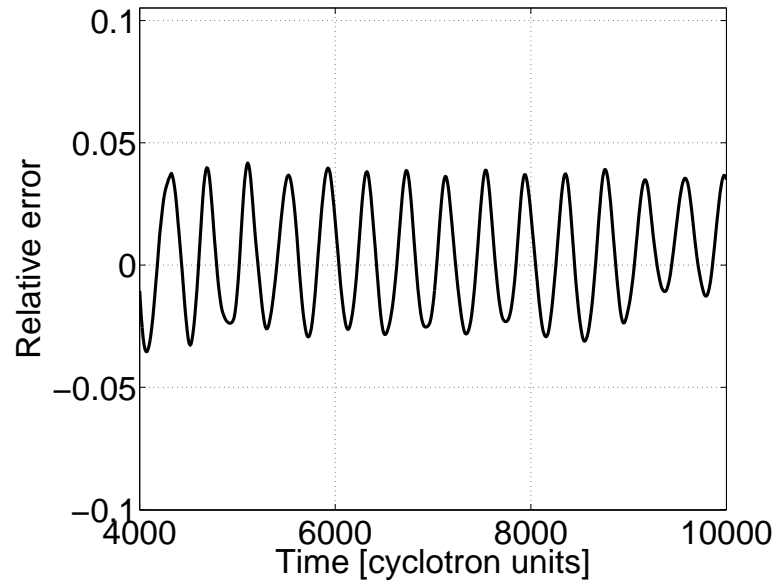

(b)

Figure 6: Time evolution of the time derivative of the kinetic energy and field energy summed over all species for $q=2$ and $n_{\mathrm{EP}} / n_{\mathrm{eq}}=0.2$ (left) and relative error (right).

Analysis of the energy exchanged between thermal/energetic species and the mode is essential to understand how the mode is excited. In figure 7 we plot the time evolution of the energy transferred from the energetic population to the mode (solid red curve), from the thermal particles to the mode (dashed blue line) and the contribution of all 
particles (dotted-dashed black line), which is the sum of energetic and thermal particle contributions, for the case $q=2$. In figure 7 a a situation at the threshold for mode excitation (see figure $3 \mathrm{~b}$ ) is given $\left(n_{\mathrm{EP}} / n_{\mathrm{th}}=0.05\right)$, whereas in figure $7 \mathrm{~b}$ a situation slightly above the threshold $\left(n_{\mathrm{EP}} / n_{\mathrm{th}}=0.1\right)$ is plotted. We can observe that, at the threshold of the instability, the energy transferred to the mode remains constant and oscillates around zero, as expected. In figure $7 \mathrm{~b}$ the situation is different. We can observe that the energetic particles give, on average, energy to the mode, whereas the thermal particles receive energy from the mode. This is of course in agreement with the standard picture of direct and inverse Landau damping, where the exciting population transfers energy to the mode and the mode in its turn transfers energy to the stabilizing population. However, it can be observed that the transferred energies oscillate, exhibiting maxima and minima, which is in agreement with the fact that both the current and the electric field are oscillating quantities at a frequency which is twice the EGAM frequency. This means in particular that thermal particles are sometimes more stabilizing and sometimes less stabilizing. Similarly, energetic particles are sometimes more destabilizing and sometimes less destabilizing. A magnified view for a given time window is provided, showing that thermal particles can actually transfer energy to the mode when the energy transferred from energetic particles to the mode exhibits a local minimum value. This occurs during the growth of the total energy transferred from particles (both thermal and energetic) to the mode. Therefore, though the global energy exchange between particles and mode could be understood as a transfer of energy from the exciting particles to the mode and from the mode to the stabilizing particles, in-depth analysis of the energy channels could provide additional information to understand more in detail the contribution of each species during the growth phase of the mode.

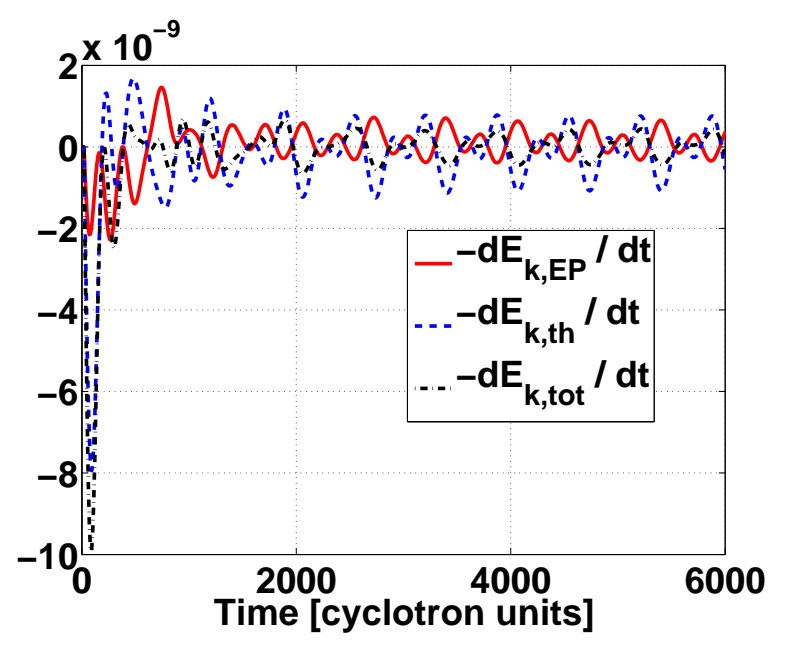

(a)

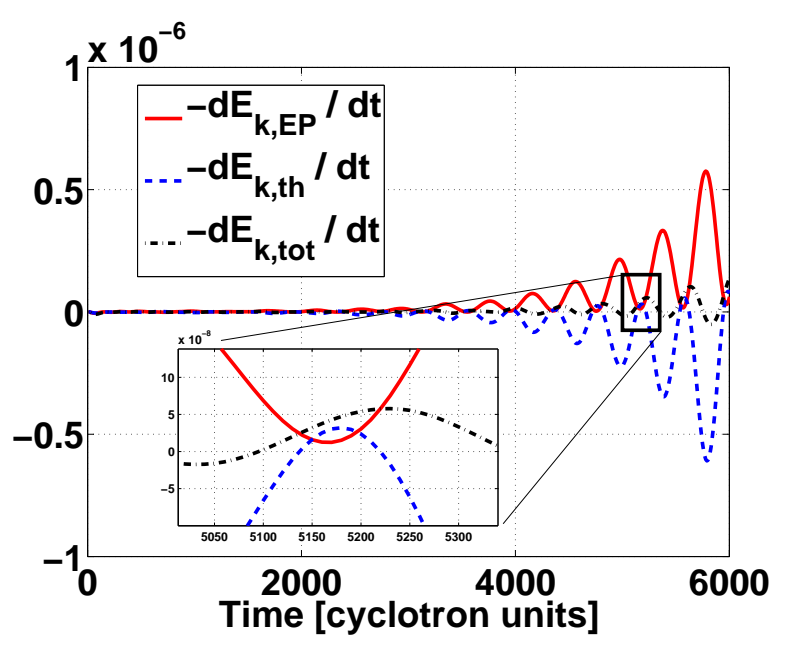

(b)

Figure 7: Time evolution of the change of the kinetic energy of the different species at $q=2$ at the threshold (left) and beyond the threshold (right). 
The energy diagnostics are also essential when analysing separately which terms in the $\mathbf{J} \cdot \mathbf{E}$ are significant for the growth of the mode, especially if one wants to determine the nature of the excited mode. The different components of the time derivative of the energetic particle kinetic energy are plotted in figure 8, following the decomposition given by expression 29. The thin solid line corresponds to the total time derivative. The thin dashed line represents the contribution of the curvature term. The thick dashed line corresponds to the contribution of the $\mu \nabla B$ term and the thick solid line gives the time evolution of the parallel velocity contribution. We can observe that the main contribution is due to the curvature, which is in agreement with the fact that we are exciting geodesic modes. However, the contribution of the curvature oscillates exhibiting maxima and minima. It is interesting to observe that although the parallel velocity contribution is overall less significant, it becomes essential when the curvature term exhibits a minimum.

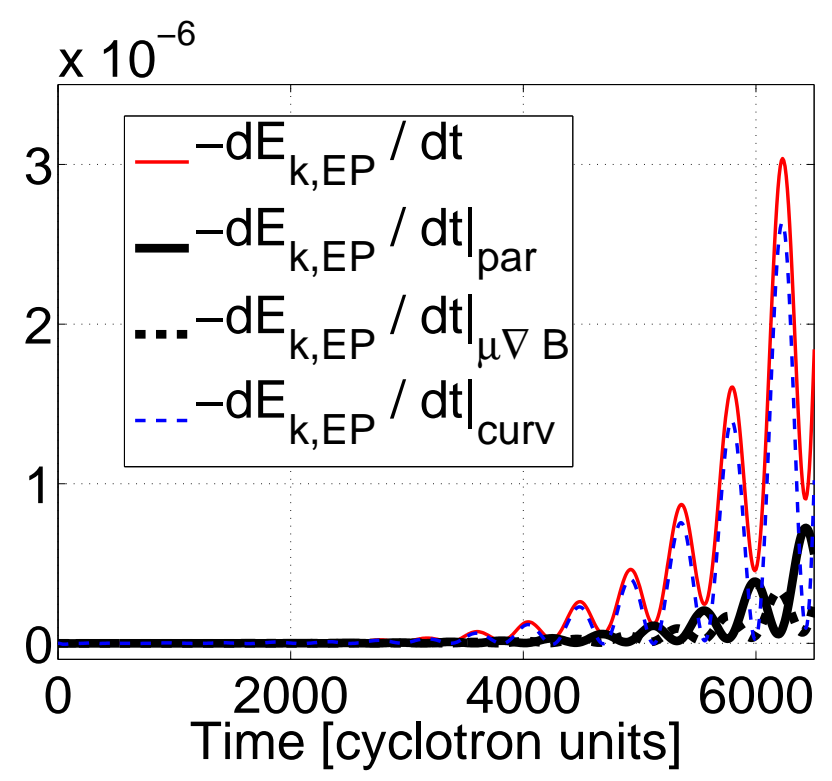

Figure 8: Comparison of two different terms to the total energy transfer from energetic particles to the mode. The fact that the main contribution comes from the curvature term indicates that the mode has indeed a geodesic nature.

\section{Conclusions and forthcoming work}

In this paper the analytic dispersion relation of geodesic acoustic modes in the presence of energetic particles is derived, for flat equilibrium profiles, neglecting FLR/FOW effects and considering the electrons as adiabatic species. The energetic particle population has been modelled with a shifted Maxwellian distribution function in the parallel velocity. This model would allow one to explain some features of energetic modes observed in experiments, such as the modification of the frequency. This model is also suitable to analyse the nature of the energetic-particle driven GAM with respect to the standard 
GAM [15] and for verification of gyrokinetic codes when the distribution function departs from a Maxwellian. It also allows us to provide a simple expression of the GAM frequency modified by energetic particles in the fluid limit. This frequency is found to increase with the pressure of energetic particles, in agreement with the kinetic prediction. The shifted Maxwellian has been implemented in the particle-in-cell code Nemorb. Simulations have been run at large $\rho_{*}$ with two values of the safety factor. In order to compare with the analytic theory, the simulations have been run linearly. The predicted frequency from the analytic theory has been recovered with linear simulations. Regarding the damping and growth rates, the effects of finite ion Larmor radius and orbit width effects have been mainly observed without energetic particles and with very low concentration of energetic particles for the higher value of the safety factor. By decreasing the ion Larmor radius for that value of safety factor, the results of the simulations show a better agreement for low concentration of energetic particles. When the energetic particle concentration is increased, decreasing the ion Larmor radius does not change significantly the growth rate of the mode, which means that these effects are less pronounced for high concentration of energetic particles. The correctness of the hypothesis of the analytic theory regarding the spatial dependence of the magnetic field has been studied with NEMORB. It has been observed that including this term affects only the initial evolution of the distribution function, but not the linear growth rate of the mode. Finally, dedicated energy diagnostics in Nemorb have been used to analyse how the energy is transferred from the particles to the mode. In particular, a multispecies code like NEMORB is suitable to decouple the energy exchanged between the mode and the thermal particles from the energy exchanged between the mode and the energetic particles. We have observed that, on average, thermal particles stabilize the mode whereas energetic particles are destabilizing. However, the instantaneous values of the energy exchange between field and particles are oscillating functions, and it is observed that the contribution of energetic and thermal particles to the excitation of the mode can be inverted within a period of oscillation. In addition, the contribution of the different terms in the particle velocity has been quantified. It has been observed that the main contribution comes from the curvature drift, which is consistent with the fact that we are exciting geodesic modes. However, this term oscillates in time and when it reaches a minimum, the parallel velocity term compensates for this loss.

For a complete description of the interaction between energetic particles and turbulence, it is essential to analyse the excitation of EGAMs also in the presence of kinetic electrons, which might contribute to the damping of the modes [22], first in the electrostatic limit by considering only trapped kinetic electrons and second in the presence of electromagnetic effects by considering fully kinetic electrons, which might broaden the spectrum and therefore enrich the interaction between EGAMs and turbulence. Therefore, forthcoming work will be presented in a different publication, where the impact of kinetic electrons is analysed with the energy diagnostics updated in order to quantify the energy transfer also in velocity space. This will be the last step before including electromagnetic effects in the excitation of geodesic acoustic modes. 


\section{Acknowledgments}

This project has received funding from the European Unions Horizon 2020 research and innovation programme under grant agreement number 633053. The views and opinions expressed herein do not necessarily reflect those of the European Commission. Simulations were performed on the IFERC-CSC Helios supercomputer within the framework of the ORBFAST project. One of the authors (D.Z.) is indebted to A. Smolyakov, for a seminal discussion which gave rise to this paper. Comments from F. Zonca and Y. Sarazin have been also kindly appreciated. One of the authors (A.B.) acknowledges useful discussions with Z. Qiu and X. Wang. Finally, the authors acknowledge fruitful discussions within the framework of the nonlinear energetic particle dynamics (NLED) European Enabling Research Project.

\section{Appendix A. Some details on the dispersion relation of energetic geodesic acoustic modes used in this paper}

In this appendix we provide the reader with the minimum details needed to obtain the dispersion relation given by expression A.37. This relation is obtained by coupling the Vlasov and the quasi-neutrality equations in the presence of adiabatic electrons and neglecting FLR/FOW effects.

\section{Appendix A.1. Vlasov equation}

The linearized gyrokinetic Vlasov equation reads $[2,5]$

$$
\left(i \omega_{t} \partial_{\theta}+\omega\right) G+-\omega_{d} \sin \theta G=-T_{\mathrm{eq}} \partial_{E} \log F_{\mathrm{eq}} \omega J_{0} \cdot \phi
$$

where $G$ is the nonadiabatic part of the perturbed distribution function, $\omega_{t}=\frac{v_{\|}}{q R}$ is the transit frequency, $\omega_{d}=k_{r} v_{d}$ is the curvature drift frequency, $T_{\text {eq }}$ a normalization temperature that we will choose equal to the bulk population temperature $\left(T_{\mathrm{eq}}=T_{\mathrm{th}}\right)$, $F_{\text {eq }}$ is the total equilibrium distribution function, $J_{0}$ is the gyro-average operator, $\phi$ is the electrostatic potential and $\theta$ is the poloidal angle. The total distribution function has been decomposed into an equilibirum part $F_{\text {eq }}$ and a perturbed part $\delta F$ as follows [5]

$$
F=F_{\text {eq }}\left(1+T_{\text {eq }} \partial_{E} \log F_{\text {eq }} J_{0} \cdot \phi+G\right)
$$

We can now project onto a Fourier basis

$$
\mathcal{S}=\mathcal{S}_{0}+\mathcal{S}_{1} \mathrm{e}^{i \theta}+\mathcal{S}_{-1} \mathrm{e}^{-i \theta}
$$

where $\mathcal{S}=\phi, G$ and consider a potential of the form $\phi_{1}=-i\left|\phi_{1}\right|$ and $\phi_{-1}=-\phi_{1}$, i.e. we keep only the up-down asymmetric components according to the GAM symmetry. In the following, $\phi_{1} \equiv\left|\phi_{1}\right|$, for the sake of clarity. As far as the distribution function is concerned, in order to keep the GAM symmetry we must impose $G_{-1}\left(v_{\|}\right)=-G_{1}\left(-v_{\|}\right)$. We now assume that the equilibrium is even in parallel velocity (no injection of 
parallel momentum) to obtain the relation between the nonadiabatic response and the electrostatic potential to write the expression of the linearized gyrokinetic Vlasov equation [5]

$$
\left(\begin{array}{l}
G_{0} \\
G_{1}
\end{array}\right)=\frac{-T_{\mathrm{eq}} \partial_{E} \log F_{\mathrm{eq}}}{\omega-\omega_{t}-\frac{\omega_{d}^{2}}{2\left(\omega+\omega_{t}\right)}}\left(\begin{array}{cc}
\omega-\omega_{t} & \frac{\omega \omega_{d}}{\omega+\omega_{t}} \\
\frac{\omega_{d}}{2} & \omega
\end{array}\right) \cdot\left(\begin{array}{c}
J_{0} \cdot \phi_{0} \\
J_{0} \cdot \phi_{1}
\end{array}\right)
$$

\section{Appendix A.2. Quasi-neutrality equation}

The quasi-neutrality equation reads

$$
\frac{e}{T_{e}}\left(\phi-\phi_{0}\right)-\frac{1}{n_{\mathrm{eq}}} \nabla_{\perp} \cdot\left(\frac{m n_{\mathrm{eq}}}{e B^{2}} \nabla_{\perp} \phi\right)=\frac{1}{n_{\mathrm{eq}}} \int \mathrm{d}^{3} \mathbf{v} J_{0} \cdot F-1
$$

where $e$ is the elementary charge, $\phi_{0}$ represents a flux-surface average, $n_{\mathrm{eq}}$ is an equilibrium density, $B$ is the magnetic field and $m$ is the ion mass. We can now set $\tau=\frac{T_{\mathrm{eq}}}{T_{e}}$ and normalize the electrostatic potential to $T_{\mathrm{eq}} / e$ in order to write

$$
\tau\left(\phi-\phi_{0}\right)-\frac{1}{n_{\mathrm{eq}}} \nabla_{\perp} \cdot\left(\frac{m n_{\mathrm{eq}} T_{\mathrm{eq}}}{e^{2} B^{2}} \nabla_{\perp} \phi\right)=\frac{1}{n_{\mathrm{eq}}} \int \mathrm{d}^{3} \mathbf{v} J_{0} \cdot F-1
$$

where $\phi$ is already the normalized electrostatic potential. Taking into account that the ion Larmor radius is $\rho_{i}=\frac{m T_{\mathrm{eq}}}{e B}$, neglecting the perpendicular gradient of the equilibrium density and performing a Fourier decomposition in the perpendicular direction, the quasi-neutrality equation reads

$$
\tau\left(\phi-\phi_{0}\right)+k_{\perp}^{2} \rho_{i}^{2} \phi=\frac{1}{n_{\text {eq }}} \int \mathrm{d}^{3} \mathbf{v} J_{0} \cdot F-1
$$

We insert the decomposition A.2 into the previous expression so that the right-hand side term can be rewritten as

$$
\frac{1}{n_{\text {eq }}} \int \mathrm{d}^{3} \mathbf{v} J_{0} \cdot F-1=\frac{1}{n_{\text {eq }}} \int \mathrm{d}^{3} \mathbf{v} J_{0} \cdot\left(F_{\text {eq }}+T_{\text {eq }} \partial_{E} F_{\text {eq }} J_{0} \cdot \phi+F_{\text {eq }} G\right)-1
$$

The gyro-average operator and the squared gyro-average operator can be expanded up to second order in $k_{r} \rho_{i}$ as follows

$$
J_{0} \approx 1-\frac{1}{2} k_{\perp}^{2} \frac{m T_{\mathrm{eq}}}{e^{2} B^{2}} \frac{\mu B}{T_{\mathrm{eq}}}, \quad J_{0}^{2} \approx 1-k_{\perp}^{2} \frac{m T_{\mathrm{eq}}}{e^{2} B^{2}} \frac{\mu B}{T_{\mathrm{eq}}}
$$

but only when applied to the electrostatic potential, which means that we neglect the perpendicular gradients of the equilibrium. Therefore, the right-hand side term reads

$$
\frac{1}{n_{\text {eq }}} \int \mathrm{d}^{3} \mathbf{v} J_{0} \cdot F-1=\frac{1}{n_{\text {eq }}} \int \mathrm{d}^{3} \mathbf{v} J_{0} \cdot T_{\text {eq }} \partial_{E} F_{\text {eq }} J_{0} \cdot \phi+\frac{1}{n_{\text {eq }}} \int \mathrm{d}^{3} \mathbf{v} J_{0} \cdot F_{\text {eq }} G
$$


and since the equilibrium gradients are neglected the gyro-average operator and $F_{\text {eq }}$ can be interchanged, so that

$$
\frac{1}{n_{\text {eq }}} \int \mathrm{d}^{3} \mathbf{v} J_{0} \cdot F-1=\frac{1}{n_{\text {eq }}} \int \mathrm{d}^{3} \mathbf{v} T_{\text {eq }} \partial_{E} F_{\text {eq }} J_{0}^{2} \cdot \phi+\frac{1}{n_{\text {eq }}} \int \mathrm{d}^{3} \mathbf{v} F_{\text {eq }} J_{0} \cdot G
$$

Taking into account the expansion of the squared gyro-average operator we get

$$
\begin{aligned}
& \frac{1}{n_{\text {eq }}} \int \mathrm{d}^{3} \mathbf{v} J_{0} \cdot F-1= \\
& \frac{1}{n_{\text {eq }}} \int \mathrm{d}^{3} \mathbf{v} T_{\text {eq }} \partial_{E} F_{\text {eq }} \phi-k_{\perp}^{2} \rho_{i}^{2} \frac{1}{n_{\text {eq }}} \int \mathrm{d}^{3} \mathbf{v} \frac{\mu B}{T_{\text {eq }}} T_{\text {eq }} \partial_{E} F_{\text {eq }} \phi+\frac{1}{n_{\text {eq }}} \int \mathrm{d}^{3} \mathbf{v} F_{\text {eq }} J_{0} \cdot G
\end{aligned}
$$

We can now make a decomposition of the equilibrium distribution function into two kinetic species: thermal particles, modelled by a centred Maxwellian distribution function $F_{\text {eq,th }}$ and energetic particles, modelled by a double shifted Maxwellian distribution function $F_{\text {eq,EP }}$. We need to calculate explicitly the derivative with respect to the energy (see equations 1 and 2)

$$
\partial_{E} F_{\text {eq }}=-\frac{1}{T_{\text {eq }}} F_{\text {eq,th }}-\frac{1}{T_{\mathrm{EP}}} F_{\text {eq,EP }}+\frac{1}{T_{\mathrm{EP}}} \tilde{F}_{\mathrm{eq}, \mathrm{EP}}
$$

where the third term reads

$$
\tilde{F}_{\text {eq,EP }}=\frac{1}{2} \frac{n_{\mathrm{EP}}}{\left(2 \pi T_{\mathrm{EP}} / m_{\mathrm{EP}}\right)^{3 / 2}} \mathrm{e}^{-\frac{\mu B}{T_{\mathrm{EP}}}} \frac{v_{0}}{v_{\|}}\left(\mathrm{e}^{-\frac{\left(v_{\|}-v_{0}\right)^{2}}{2 T_{\mathrm{EP}} / m_{\mathrm{EP}}}}-\mathrm{e}^{-\frac{\left(v_{\|}+v_{0}\right)^{2}}{2 T_{\mathrm{EP}} / m_{\mathrm{EP}}}}\right)
$$

From now on we will assume that $T_{\mathrm{EP}}=T_{\mathrm{th}}$. The right-hand side term reads

$$
\frac{1}{n_{\mathrm{eq}}} \int \mathrm{d}^{3} \mathbf{v} J_{0} \cdot F-1=-\left(1-\langle 1\rangle_{\mathrm{EP}}\right) \phi+\left(1-\langle 1\rangle_{\mathrm{EP}}\right) k_{\perp}^{2} \rho_{i}^{2} \phi+\left\langle J_{0} \cdot G\right\rangle_{F_{\mathrm{eq}}}
$$

where the averages $\langle\cdots\rangle_{F_{\mathrm{eq}}}$ and $\langle\cdots\rangle_{\mathrm{EP}}$ are defined as

$$
\begin{gathered}
\langle\cdots\rangle_{F_{\mathrm{eq}}}=\frac{1}{n_{\mathrm{eq}}} \int \mathrm{d}^{3} \mathbf{v} \cdots F_{\mathrm{eq}} \\
\langle\cdots\rangle_{\mathrm{EP}}=\frac{1}{n_{\mathrm{eq}}} \int \mathrm{d}^{3} \mathbf{v} \cdots \tilde{F}_{\mathrm{eq}, \mathrm{EP}}
\end{gathered}
$$

with $F_{\text {eq }}=F_{\text {eq,th }}+F_{\text {eq,EP. }}$. The term $k_{\perp}^{2} \rho_{i}^{2} \phi$ cancels with the same term on the left-hand side of the quasi-neutrality equation. Therefore, the quasineutrality equation can be rewritten as follows

$$
\tau\left(\phi-\phi_{0}\right)=-\left(1-\langle 1\rangle_{\mathrm{EP}}\right) \phi-\langle 1\rangle_{\mathrm{EP}} k_{\perp}^{2} \rho_{i}^{2} \phi+\left\langle J_{0} \cdot G\right\rangle_{F_{\mathrm{eq}}}
$$

Taking into account the projection onto a Fourier basis, the quasineutrality equation reads

$$
\left\langle\left(\begin{array}{c}
J_{0} \cdot G_{0} \\
J_{0} \cdot G_{1}
\end{array}\right)\right\rangle_{F_{\mathrm{eq}}}=\left(\begin{array}{cc}
1-\langle 1\rangle_{\mathrm{EP}}\left(1-k_{\perp}^{2} \rho_{i}^{2}\right) & 0 \\
0 & (1+\tau)-\langle 1\rangle_{\mathrm{EP}}\left(1-k_{\perp}^{2} \rho_{i}^{2}\right)
\end{array}\right)\left(\begin{array}{c}
\phi_{0} \\
\phi_{1}
\end{array}\right)
$$


Appendix A.3. Combining Vlasov and Quasineutrality equations

We combine equations A.4 and A.19 to obtain the dispersion relation A.37 that we give at the beginning of this paper. For this purpose we first insert equation A.4 on the left-hand side of equation A.19 to eliminate $G_{0}$ and $G_{1}$. As explained earlier, the gyro-average operator acts only on the electrostatic potential. We can write the quasineutrality equation in terms only of the electrostatic potential as follows

$$
\mathrm{A} \cdot \boldsymbol{\Phi}=0
$$

where

$$
\Phi=\left(\begin{array}{c}
\phi_{0} \\
\phi_{1}
\end{array}\right)
$$

and $\mathbf{A}$ is an operator proportional to the squared gyro-average operator, written in tensor form as

$$
\mathbf{A}=\left(\begin{array}{ll}
A_{11} & A_{12} \\
A_{21} & A_{22}
\end{array}\right)
$$

The elements of $\mathbf{A}$ are

$$
\begin{aligned}
& A_{11}=1-\langle 1\rangle_{\mathrm{EP}}\left(1-k_{\perp}^{2} \rho_{i}^{2}\right)+\left\langle\frac{T_{\mathrm{eq}} \partial_{E} \log F_{\mathrm{eq}}}{\omega-\omega_{t}-\frac{\omega_{d}^{2}}{2\left(\omega+\omega_{t}\right)}}\left(\omega-\omega_{t}\right) J_{0}^{2}\right\rangle_{F_{\mathrm{eq}}} \\
& A_{12}=\left\langle\frac{T_{\mathrm{eq}} \partial_{E} \log F_{\mathrm{eq}}}{\omega-\omega_{t}-\frac{\omega_{d}^{2}}{2\left(\omega+\omega_{t}\right)}} \frac{\omega \omega_{d}}{\omega+\omega_{t}} J_{0}^{2}\right\rangle_{F_{\mathrm{eq}}} \\
& A_{21}=\left\langle\frac{T_{\mathrm{eq}} \partial_{E} \log F_{\mathrm{eq}}}{\omega-\omega_{t}-\frac{\omega_{d}^{2}}{2\left(\omega+\omega_{t}\right)}} \frac{\omega_{d}}{2} J_{0}^{2}\right\rangle_{F_{\mathrm{eq}}} \\
& A_{22}=1+\tau-\langle 1\rangle_{\mathrm{EP}}\left(1-k_{\perp}^{2} \rho_{i}^{2}\right)+\left\langle\frac{T_{\mathrm{eq}} \partial_{E} \log F_{\mathrm{eq}}}{\omega-\omega_{t}-\frac{\omega_{d}^{2}}{2\left(\omega+\omega_{t}\right)}} \omega J_{0}^{2}\right\rangle_{F_{\mathrm{eq}}}
\end{aligned}
$$

Appendix A.3.1. Term $A_{11}$ The term in between $\langle\cdots\rangle_{F_{\mathrm{eq}}}$ brackets in the element A.23 can be approximated by extracting the difference $\omega-\omega_{t}$ in the denominator, which simplifies with $\omega-\omega_{t}$ that multiplies the squared gyro-average operator. We then perform an expansion of $\left(1-\frac{\omega_{d}^{2}}{2\left(\omega^{2}-\omega_{t}^{2}\right)}\right)^{-1}$ up to second order in $k_{\perp}$. This term is therefore written as

$$
T_{\text {eq }} \partial_{E} \log F_{\text {eq }} J_{0}^{2}+\frac{\omega_{d}^{2}}{2\left(\omega^{2}-\omega_{t}^{2}\right)} T_{\text {eq }} \partial_{E} \log F_{\text {eq }}
$$

when applying the operator $\langle\cdots\rangle_{F_{\text {eq }}}$ and the expansion of $J_{0}^{2}$ one gets

$$
-1+\langle 1\rangle_{\mathrm{EP}}\left(1-k_{\perp}^{2} \rho_{i}^{2}\right)+k_{\perp}^{2} \rho_{i}^{2}-\frac{1}{2}\left\langle\frac{\omega_{d}^{2}}{\omega^{2}-\omega_{t}^{2}}\right\rangle_{F_{\mathrm{eq}}}+\frac{1}{2}\left\langle\frac{\omega_{d}^{2}}{\omega^{2}-\omega_{t}^{2}}\right\rangle_{\mathrm{EP}}
$$


Analytic theory and NEMORB simulations of EGAMs

The first two terms cancel with the first two terms of $A_{11}$. Therefore, we can write

$$
A_{11} \approx\left(1-\frac{1}{2} \frac{1}{\rho_{i}^{2}}\left\langle\frac{v_{d}^{2}}{\omega^{2}-\omega_{t}^{2}}\right\rangle_{F_{\mathrm{eq}}}+\frac{1}{2} \frac{1}{\rho_{i}^{2}}\left\langle\frac{v_{d}^{2}}{\omega^{2}-\omega_{t}^{2}}\right\rangle_{\mathrm{EP}}\right) k_{\perp}^{2} \rho_{i}^{2}
$$

Appendix A.3.2. Term $A_{12}$ We take again the term $\omega-\omega_{t}$ out of the denominator and perform the same expansion as in the previous case up to second order in $k_{\perp}$. We can also write

$$
\frac{\omega \omega_{d}}{\omega^{2}-\omega_{t}^{2}}=\frac{1}{2}\left(\frac{\omega_{d}}{\omega-\omega_{t}}+\frac{\omega_{d}}{\omega+\omega_{t}}\right)
$$

When applying the average $\langle\cdots\rangle_{F_{\text {eq }}}$ and making use of the parity of the equilibrium in $v_{\|}$we can write

$$
\left\langle T_{\text {eq }} \partial_{E} \log F_{\text {eq }} \frac{\omega \omega_{d}}{\omega^{2}-\omega_{t}^{2}}\right\rangle_{F_{\text {eq }}}=\left\langle T_{\text {eq }} \partial_{E} \log F_{\text {eq }} \frac{\omega_{d}}{\omega-\omega_{t}}\right\rangle_{F_{\text {eq }}}
$$

Therefore, this element of the array reads

$$
A_{12} \approx-\left(\frac{1}{\rho_{i}}\left\langle\frac{v_{d}}{\omega-\omega_{t}}\right\rangle_{F_{\mathrm{eq}}}-\frac{1}{\rho_{i}}\left\langle\frac{v_{d}}{\omega-\omega_{t}}\right\rangle_{\mathrm{EP}}\right) k_{\perp} \rho_{i}
$$

Appendix A.3.3. Term $A_{21}$ In a similar way as we did for the previous term, it is straightforward to prove that

$$
A_{21} \approx-\frac{1}{2}\left(\frac{1}{\rho_{i}}\left\langle\frac{v_{d}}{\omega-\omega_{t}}\right\rangle_{F_{\mathrm{eq}}}-\frac{1}{\rho_{i}}\left\langle\frac{v_{d}}{\omega-\omega_{t}}\right\rangle_{\mathrm{EP}}\right) k_{\perp} \rho_{i}
$$

Appendix A.3.4. Term $A_{22}$ Performing the same expansions as in the previous terms, we can write

$$
A_{22} \approx 1+\tau-\langle 1\rangle_{\mathrm{EP}}-\left\langle\frac{\omega}{\omega-\omega_{t}}\right\rangle_{F_{\mathrm{eq}}}+\left\langle\frac{\omega}{\omega-\omega_{t}}\right\rangle_{\mathrm{EP}}
$$

Appendix A.4. Obtaining the final expression for the dispersion relation

The system A.20 has a non trivial solution if and only if $\operatorname{det} \mathbf{A}=0$, which is expressed as

$$
\begin{aligned}
\left(1-\frac{1}{2} \frac{1}{\rho_{i}^{2}}\left\langle\frac{v_{d}^{2}}{\omega^{2}-\omega_{t}^{2}}\right\rangle_{F_{\mathrm{eq}}}+\frac{1}{2} \frac{1}{\rho_{i}^{2}}\left\langle\frac{v_{d}^{2}}{\omega^{2}-\omega_{t}^{2}}\right\rangle_{\mathrm{EP}}\right) \\
\times\left(1+\tau-\langle 1\rangle_{\mathrm{EP}}-\left\langle\frac{\omega}{\omega-\omega_{t}}\right\rangle_{F_{\mathrm{eq}}}+\left\langle\frac{\omega}{\omega-\omega_{t}}\right\rangle_{\mathrm{EP}}\right) \\
-\frac{1}{2} \frac{1}{\rho_{i}^{2}}\left(\left\langle\frac{v_{d}}{\omega-\omega_{t}}\right\rangle_{F_{\mathrm{eq}}}-\left\langle\frac{v_{d}}{\omega-\omega_{t}}\right\rangle_{\mathrm{EP}}\right)^{2}=0
\end{aligned}
$$


We can now make use of the expression of the curvature drift velocity

$$
v_{d} \approx \frac{m v_{\|}^{2}+\mu B}{e B R_{0}}
$$

and integrate over $\mu$ to find the final dispersion relation given in the main text of the paper

$$
\begin{aligned}
\mathcal{D}(\Omega)=\{1- & \left.\frac{1}{2}\left\langle\frac{\zeta^{4}+2 \zeta^{2}+2}{\Omega^{2}-\zeta^{2} / q^{2}}\right\rangle_{F_{\mathrm{eq}}}+\frac{1}{2}\left\langle\frac{\zeta^{4}+2 \zeta^{2}+2}{\Omega^{2}-\zeta^{2} / q^{2}}\right\rangle_{\mathrm{EP}}\right\} \\
& \times\left\{1+\tau-\langle 1\rangle_{\mathrm{EP}}-\left\langle\frac{\Omega}{\Omega-\zeta / q}\right\rangle_{F_{\mathrm{eq}}}+\left\langle\frac{\Omega}{\Omega-\zeta / q}\right\rangle_{\mathrm{EP}}\right\} \\
& -\frac{1}{2}\left\{\left\langle\frac{\zeta^{2}+1}{\Omega-\zeta / q}\right\rangle_{F_{\mathrm{eq}}}-\left\langle\frac{\zeta^{2}+1}{\Omega-\zeta / q}\right\rangle_{\mathrm{EP}}\right\}^{2}=0
\end{aligned}
$$

where $\Omega=\omega R / v_{\mathrm{th}}, R$ is the major radius of the tokamak and the brackets represent the following averages in velocity space

$$
\begin{gathered}
\langle\cdots\rangle_{F_{\text {eq }}}=\frac{1}{\sqrt{2 \pi}} \int d \zeta\{\cdots\} \sum_{\eta=0, \pm 1} N_{\eta} e^{\frac{(\zeta-\eta \bar{\zeta})^{2}}{2}} \\
\langle\cdots\rangle_{\mathrm{EP}}=\frac{1}{\sqrt{2 \pi}} \int d \zeta\{\cdots\} \frac{\bar{\zeta}}{\zeta} \sum_{\eta= \pm 1} \eta N_{\eta} e^{-\frac{(\zeta-\eta \bar{\zeta})^{2}}{2}}
\end{gathered}
$$

where $N_{0}=\frac{n_{\mathrm{eq}}-n_{\mathrm{EP}}}{n_{\mathrm{eq}}}, N_{ \pm 1}=\frac{n_{\mathrm{EP}}}{2 n_{\mathrm{eq}}}$ account for the concentrations of thermal and energetic particles, respectively. The parallel velocities $v_{\|}$and $v_{0}$ are normalized to the thermal velocity $v_{\text {th }}=\sqrt{T_{\text {th }} / m_{\text {th }}}$ and rewritten as $\zeta$ and $\bar{\zeta}$, respectively. Note that this time the brackets represent averages in parallel velocity space, since a first integration over $\mu$ has already be performed.

\section{Appendix B. Dispersion relation in the hydrodynamic limit}

The hydrodynamic limit is defined as $q \Omega \gg 1$. Assuming this limit in the dispersion relation, it is possible to expand the denominators containing the resonance by neglecting the second order in $q^{-1} \Omega^{-1}$

$$
\begin{gathered}
\frac{1}{\Omega^{2}-\zeta^{2} / q^{2}} \approx \frac{1}{\Omega^{2}} \\
\frac{1}{\Omega-\zeta / q} \approx \frac{1}{\Omega}\left(1+\frac{\zeta}{q \Omega}\right)
\end{gathered}
$$

Taking into account that the brackets $\langle\cdots\rangle_{F_{\mathrm{eq}}}$ and $\langle\cdots\rangle_{\mathrm{EP}}$ vanish for odd functions, the term proportional to $\zeta$ does not contribute. The brackets $\langle\cdots\rangle_{F_{\text {eq }}}$ give for the different terms

$$
\left\langle\frac{\zeta^{4}+2 \zeta^{2}+2}{\Omega^{2}-\zeta^{2} / q^{2}}\right\rangle_{F_{\mathrm{eq}}} \approx \frac{1}{\Omega^{2}}\left[3+4 \bar{\chi}^{2} \frac{n_{\mathrm{EP}}}{n_{\mathrm{eq}}}\left(3+\bar{\chi}^{2}\right)+2\left(1+2 \bar{\chi}^{2} \frac{n_{\mathrm{EP}}}{n_{\mathrm{eq}}}\right)+2\right]
$$




$$
\begin{gathered}
\left\langle\frac{\Omega}{\Omega-\zeta / q}\right\rangle_{F_{\mathrm{eq}}} \approx 1 \\
\left\langle\frac{\zeta^{2}+1}{\Omega-\zeta / q}\right\rangle_{F_{\mathrm{eq}}} \approx \frac{1}{\Omega}\left(2+2 \bar{\chi}^{2} \frac{n_{\mathrm{EP}}}{n_{\mathrm{eq}}}\right)
\end{gathered}
$$

where $\bar{\chi}=\bar{\zeta} / \sqrt{2}$. To calculate the brackets $\langle\cdots\rangle_{\mathrm{EP}}$ we neglect the terms proportional to $\langle 1\rangle_{\mathrm{EP}}$. This is justified for energetic particles exhibiting sufficiently high mean velocity $\bar{\zeta}$, which is the case in this paper. Therefore, we have

$$
\begin{gathered}
\langle 1\rangle_{\mathrm{EP}} \approx 0 \\
\left\langle\frac{\zeta^{4}+2 \zeta^{2}+2}{\Omega^{2}-\zeta^{2} / q^{2}}\right\rangle_{\mathrm{EP}} \approx \frac{4}{\Omega^{2}} \frac{n_{\mathrm{EP}}}{n_{\mathrm{eq}}} \bar{\chi}^{2}\left(\frac{5}{2}+\bar{\chi}^{2}\right) \\
\left\langle\frac{\Omega}{\Omega-\zeta / q}\right\rangle_{\mathrm{EP}} \approx 0 \\
\left\langle\frac{\zeta^{2}+1}{\Omega-\zeta / q}\right\rangle_{\mathrm{EP}} \approx \frac{2 \bar{\chi}^{2}}{\Omega} \frac{n_{\mathrm{EP}}}{n_{\mathrm{eq}}}
\end{gathered}
$$

Inserting these expressions into the dispersion and multiplying by $\Omega^{2}$, we get

$$
\Omega^{2}\left[1-\frac{1}{\Omega^{2}}\left(\frac{7}{2}+3 \frac{n_{\mathrm{EP}}}{n_{\mathrm{eq}}} \bar{\chi}^{2}\right)\right] \approx 2
$$

And the GAM frequency modified by the presence of energetic particles finally reads

$$
\Omega_{\mathrm{GAM}, \mathrm{EP}} \approx \sqrt{\Omega_{\mathrm{GAM}}^{2}+3 \frac{n_{\mathrm{EP}}}{n_{\mathrm{eq}}} \bar{\chi}^{2}}
$$

[1] GY Fu. Phys. Rev. Lett., 101(18):185002, 2008.

[2] Z. Qiu, F. Zonca, and L. Chen. Plasma Phys. Control. Fusion, 52:095003, 2010.

[3] CJ Boswell, HL Berk, DN Borba, T. Johnson, SD Pinches, and SE Sharapov. Physics Letters A, 358(2):154-158, 2006.

[4] R. Nazikian, GY Fu, ME Austin, HL Berk, RV Budny, NN Gorelenkov, WW Heidbrink, CT Holcomb, GJ Kramer, GR McKee, et al. Phys. Rev. Lett., 101(18):185001, 2008.

[5] D. Zarzoso, X. Garbet, Y. Sarazin, R. Dumont, and V. Grandgirard. Phys. Plasmas, 19(2):022102022102, 2012.

[6] D Zarzoso, Y Sarazin, X Garbet, R Dumont, A Strugarek, J Abiteboul, T Cartier-Michaud, G DifPradalier, Ph Ghendrih, V Grandgirard, et al. Phys. Rev. Lett., 110(12):125002, 2013.

[7] F. Zonca, L. Chen, and Z. Qiu. In Fusion Energy 2008 (Proc. 22nd Int. Conf. Geneva, 2008) (Vienna: IAEA) CD-ROM file.

[8] N. Winsor, J.L. Johnson, and J.M. Dawson. Phys. Fluids, 11:2448, 1968.

[9] S Jolliet, A Bottino, P Angelino, R Hatzkyc, TM Trana, BF Mcmillan, O Sauter, K Appert, Y Idomura, and L Villard. Comp. Phys. Comm., 177(5):409-425, 2007.

[10] A Bottino, Thibaut Vernay, B Scott, S Brunner, R Hatzky, Sébastien Jolliet, BF McMillan, TM Tran, and L Villard. Plasma Phys. Control. Fusion, 53(12):124027, 2011. 
[11] F Zonca, L Chen, and R A Santoro. Plasma Phys. Control. Fusion, 38(11):2011, 1996.

[12] A typo in ref. 5 was found during the redaction of this paper. note that the sum of plasma dispersion functions in the definition of $Z_{1}$ has to be changed into one single plasma dispersion function.

[13] F. Zonca and L. Chen. Radial structures and nonlinear excitation of geodesic acoustic modes. EPL (Europhysics Letters), 83:35001, 2008.

[14] Ph Lauber, M Schneller, A Biancalani, D Zarzoso, S Guenter, I Classen, M Maraschek, Sylvie da Graca, V Igochine, G Tardini, B Geiger, G Conway, P Simon, J Hobirk, and ASDEX Upgrade Team. Off-axis nbi-driven modes at asdex upgrade. In 13th IAEA TCM on Energetic Particles, Beijing, September 2013).

[15] JB Girardo, D Zarzoso, R Dumont, X Garbet, Y Sarazin, and S Sharapov. Link between energetic and standard geodesic acoustic modes. submitted to Phys. Plasmas, 2014.

[16] AJ Brizard and TS Hahm. Rev. Mod. Phys., 79(2):421, 2007.

[17] A Biancalani, A Bottino, Ph Lauber, and D Zarzoso. Nuclear Fusion, 54, 2014.

[18] H Sugama and T-H Watanabe. Journal of plasma physics, 74:139-140, 2008.

[19] A Bottino, AG Peeters, O Sauter, J Vaclavik, L Villard, and ASDEX Upgrade Team. Phys. Plasmas (1994-present), 11(1):198-206, 2003.

[20] TS Hahm. Phys. Plasmas (1994-present), 3(12):4658-4664, 1996.

[21] B Scott and J Smirnov. Phys. Plasmas (1994-present), 17(11):112302, 2010.

[22] HS Zhang and Z Lin. Phys. Plasmas, 17:072502, 2010. 